\title{
Magnetic incommensurability in a doped Mott insulator
}

\author{
Z. Y. Weng, D. N. Sheng, and C. S. Ting \\ Texas Center for Superconductivity and Department of Physics, University of Houston, Houston, Texas 77204-5932
}

(Received 22 September 1998)

\begin{abstract}
In this paper we explore the incommensurate spatial modulation of spin-spin correlations as the intrinsic property of the doped Mott insulator, described by the $t-J$ model. We show that such an incommensurability is a direct manifestation of the phase-string effect introduced by doped holes in both one- and two-dimensional cases. The magnetic incommensurate peaks of dynamic spin susceptibility in momentum space are in agreement with the neutron-scattering measurement of cuprate superconductors in both position and doping dependence. In particular, this incommensurate structure can naturally reconcile the neutron-scattering and NMR experiments of cuprates. [S0163-1829(99)03017-9]
\end{abstract}

\section{INTRODUCTION}

Antiferromagnetic (AF) spin correlations in cuprates persist into the metallic phase as evidenced, for example, in neutron-scattering measurements. Compared to the insulating phase, however, a qualitative change in the nature of $\mathrm{AF}$ correlations has also been observed. ${ }^{1-5}$ The original magnetic peak in the dynamic spin susceptibility centered at momentum $(\pi, \pi)$ splits into four incommensurate peaks at $(\pi \pm \pi \bar{\delta}, \pi)$ and $(\pi, \pi \pm \pi \bar{\delta})$ in the metallic or superconducting regime, as has been found in the $\mathrm{La}-\mathrm{Sr}-\mathrm{Cu}-\mathrm{O}$ compounds $^{1-4}$ (a recent measurement ${ }^{5}$ indicates a similar structure for $\mathrm{Y}-\mathrm{Ba}-\mathrm{Cu}-\mathrm{O}$ compounds as well). The incommensurability is insensitive to both energy and temperature, corresponding to a spatial modulation of spin-spin correlations in real space.

The incommensurability $\bar{\delta}$ has been identified to be proportional to doping concentration $\delta$ in approximately a universal fashion: $\bar{\delta} \simeq 2 \delta \delta^{2,4}$ This observation poses a critical challenge to theories of incommensurability based on the Fermi surface topology: ${ }^{6-8}$ since the Fermi surface encloses a $1-\delta$ amount of electrons according to the Luttinger theorem, it is not a trivial issue why the doping concentration $\delta$ of holes should dictate the incommensurability. A very similar issue has already been well known in the transport properties of cuprates where charge carriers are always characterized by the hole nature with the total number determined by $\delta$ despite a large Fermi surface. This phenomenon has been interpreted due to the existence of a doped-Mott insulator ${ }^{9}$ because of the strong Coulomb interaction among electrons. With striking similarity, magnetic incommensurability may be another intrinsic consequence of the doped-Mott insulator.

A further challenge to any theory of magnetic incommensurability comes from NMR spin-relaxation rates $1 / T_{1}$. As pointed out by Walstedt, Shastry, and Cheong, ${ }^{10}$ if the nonKorringa behavior of $1 / T_{1}$ for planar ${ }^{63} \mathrm{Cu}$ nuclear spins - a hallmark of normal-state anomalies of cuprates - originates from the AF correlations, this effect may generally "leak" to the planar ${ }^{17} \mathrm{O}$ nuclear spins if the AF correlations become incommensurate. Thus the non-Korringa behavior would have to show up in the temperature dependence of $1 /{ }^{17} T_{1}$.
This was indeed predicted by theories ${ }^{6-8}$ where the incommensurability is interpreted in terms of the Fermi-surface topology. But experimentally the "leakage" of nonKorringa behavior to ${ }^{17} \mathrm{O}$ spins has never been observed, ${ }^{10}$ and the temperature behavior of $1 /{ }^{17} T_{1}$ is drastically different from that of $1 /{ }^{63} T_{1}$, implying a near-perfect commensurate AF correlations within a one-component-theory framework. So how to reconcile the NMR and neutron measurements remains a serious puzzle in constructing a consistent microscopic theory.

In this paper, we present a theoretical understanding of spatially modulated spin-spin correlations as a result of the doped Mott insulator described by the $t-J$ model. Such a Mott insulator at half-filling is an antiferromagnet whose magnetic properties have been well understood. ${ }^{11,12} \mathrm{~A}$ hole doped into such an antiferromagnet will induce a phasestring "defect" in the spin background which is nonrepairable at low energy. ${ }^{13}$ It represents a singular doping effect and profoundly modifies both spin and charge correlations in the presence of finite density of doped holes. ${ }^{14}$ At lowdoping where the "rigidity" of the half-filled AF state still persists, described by a bosonic resonating valence bond (RVB) pairing, ${ }^{15,16}$ a mean-field theory ${ }^{17}$ incorporating the phase-string effect is able to combine the AF insulating phase, superconducting phase, and an anomalous normal state within a unified phase diagram naturally. In the present paper we demonstrate that it is in this regime that a spatial modulation of spin-spin correlations indeed occurs as a direct manifestation of the nonrepairable phase string effect. Such an incommensurability is consistent with the experimental measurements in cuprate superconductors. In particular, such a mechanism for spatial modulation can uniquely reconcile the above-mentioned neutron and NMR experiments in cuprates.

The present work will be based on the so-called phasestring formalism ${ }^{14}$ of the $t-J$ model in which the phase-string effect is explicitly incorporated into the Hamiltonian. In this formalism, the spin raising operator $S_{i}^{+}$reads

$$
S_{i}^{+}=b_{i \uparrow}^{\dagger} b_{i \downarrow}(-1)^{i} e^{i \Phi_{i}^{h}} .
$$

Here $b_{i \sigma}$ denotes a bosonic spinon annihilation operator. Compared to the conventional Schwinger-boson 


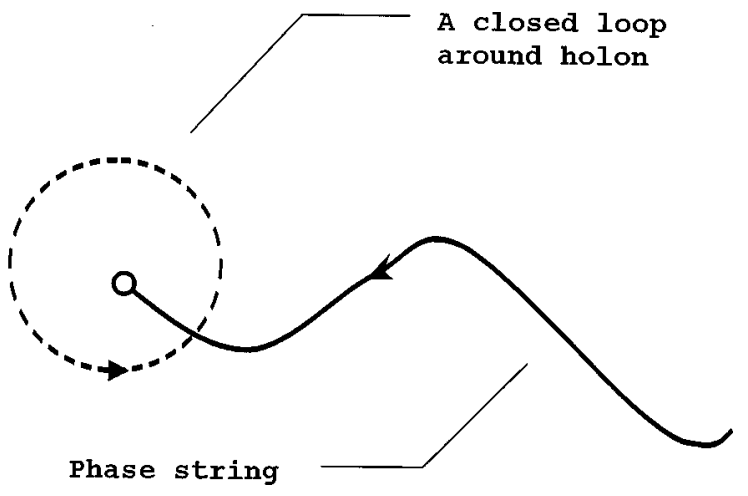

FIG. 1. The topology of a holon due to the phase string: any closed loop around it will cut through the phase string once or an odd number of times.

formalism, ${ }^{16}$ a distinctive feature is the phase $\Phi_{i}^{h}$ which is defined by

$$
\Phi_{i}^{h} \equiv \sum_{l \neq i} \operatorname{Im} \ln \left(z_{i}-z_{l}\right) n_{l}^{h} .
$$

It describes a phase vortex structure centered on holons: $n_{l}^{h}$ is the holon number operator at site $l$ and $z_{i}=x_{i}+i y_{i}$ represents the complex coordinate of a lattice site $i$. If one changes $z_{i}$ continuously around a holon site $l$ once, $\Phi_{i}^{h}$ will gain an additional $2 \pi$ phase. Such a vorticity around a holon reflects the topology of the phase-string effect as schematically illustrated in Fig. 1. In this way, spins become nonlocally dependent on the positions of holes and the doping effect explicitly enters spin-spin correlations through $\Phi_{i}^{h}$, in addition to a modification of spinon spectrum via the Hamiltonian.

In Sec. II, we show that an incommensurate spatial oscillation because of $\Phi_{i}^{h}$ will modulate the original commensurate oscillation of $(-1)^{i}$ in Eq. (1) at finite doping in both one-dimensional (1D) and two-dimensional (2D) cases. The incommensurability in 1D recovers the well-known Luttinger-liquid behavior, while in 2D it explains the experimental results in cuprates including the incommensurate peak positions in momentum space and the doping dependence of the incommensurability. We then further show that the effect of such a spatial modulation of spin-spin correlations cannot be picked up by NMR spin-relaxation rates precisely due to its singular nature associated with doped holes, and thus reconcile the neutron and NMR experiments in cuprates. Finally, a conclusive discussion is presented in Sec. III.

\section{INCOMMENSURABILITY DUE TO PHASE-STRING EFFECT}

According to Eq. (1), the transverse spin-spin correlation function can be written down as follows:

$$
\left\langle S_{i}^{+}(t) S_{j}^{-}(0)\right\rangle=(-1)^{i-j}\left\langle b_{i \uparrow}^{\dagger}(t) b_{i \downarrow}(t) \Pi_{i j}^{h}(t) b_{j \downarrow}^{\dagger}(0) b_{j \uparrow}(0)\right\rangle,
$$

where

$$
\Pi_{i j}^{h}(t) \equiv e^{i \Phi_{i}^{h}(t)} e^{-i \Phi_{j}^{h}(0)} .
$$

(The longitudinal correlation function will be discussed in the Appendix.) At half-filling, $\Phi_{i}^{h}=0$ and the prefactor $(-1)^{i-j}$ in Eq. (3) determines a commensurate AF oscillation of the spin-spin correlation function: $(-1)^{i-j}$ $\equiv e^{i \mathbf{Q}_{0} \cdot\left(\mathbf{r}_{i}-\mathbf{r}_{j}\right)}$ with the AF wave vector

$$
\mathbf{Q}_{0}=( \pm \pi / a, \pm \pi / a),
$$

where $a$ is the lattice constant. [Note that the average in Eq. (3) will also produce a (small) term which oscillates as $(-1)^{i-j}$ in real space and compensates the prefactor in Eq. (3), leading to a ferromagnetic component of spin-spin correlations at half-filling. ${ }^{16}$ ]

At finite doping, the topological phase $\Phi_{i}^{h}$ defined in Eq. (2) will qualitatively modify the spatial modulation of the AF spin-spin correlations. For the purpose of illustration, in the following we discuss the 1D case first as an example, where the phase string effect has been already shown ${ }^{14}$ to play a crucial role responsible for Luttinger-liquid behavior. Then we discuss the 2D case and make the comparison with experiment.

\section{A. Incommensurability in 1D}

In $1 \mathrm{D}$ case, $\Phi_{i}^{h}$ simply reduces to ${ }^{14}$

$$
\Phi_{i}^{h}= \pm \pi \sum_{l>i} n_{l}^{h}
$$

Let us consider the equal-time case first, where

$$
\Pi_{i j}^{h}=e^{i\left(\Phi_{i}^{h}-\Phi_{j}^{h}\right)}=e^{ \pm i \pi \sum_{i<l<j} n_{l}^{h} .}
$$

Here we assume $i<j$ without loss of generality. Expression (7) clearly shows that every holon sitting between $i$ and $j$ will contribute a $\pi$ phase shift to $\Pi_{i j}^{h}$.

In the $1 \mathrm{D}$ case, one may make ${ }^{14}$ a simple mean-field decoupling of the spinon and holon degrees of freedom defined in the present phase-string representation. Due to the phasestring effect, a mean-field decoupling in other forms of decomposition like the slave-boson and slave-fermion representations should not be appropriate. We assume that the mean-field decoupling of spinon and holon in the present representation corresponds to the well-known spin-charge separation in this model and get the correct asymptotic behavior of various correlation functions ${ }^{14}$ without considering fluctuations beyond the mean-field state, which in turn suggests that the phase-string effect is indeed the only crucial phase fluctuations for low-energy, long-wavelength physics. In the following we show how the magnetic incommensurability is produced by the phase string effect in this mean-field treatment.

Due to the spinon-holon mean-field decoupling, one may take separate averages over spinon and holon in Eq. (3). Define $N_{i j}^{h}=\Sigma_{i<l<j} n_{l}^{h}$. The average of $\Pi_{i j}^{h}$ over holon degrees of freedom can be evaluated as follows:

$$
\left\langle\Pi_{i j}^{h}\right\rangle=e^{ \pm i \pi\left\langle N_{i j}^{h}\right\rangle}\left\langle e^{ \pm i \pi\left[N_{i j}^{h}-\left\langle N_{i j}^{h}\right\rangle\right]}\right\rangle=e^{i \delta Q\left(x_{i}-x_{j}\right)}
$$

$\times$ slow oscillation part

with 


$$
\delta Q\left(x_{i}-x_{j}\right) \equiv \pm \pi\left\langle N_{i j}^{h}\right\rangle .
$$

To leading approximation, $\Pi_{i j}^{h}$ will then contribute to an $a d$ ditional oscillation factor at wave vector

$$
\delta Q= \pm \pi \delta / a
$$

Besides such a singular oscillating part, there is a slow oscillation part in Eq. (8) which only gives rise to an additional decay, determined at $J \rightarrow 0^{+}$limit as ${ }^{14} \sim 1 /\left|x_{i j}\right|^{1 / 2}$ at $\left|x_{i j}\right|$ $\equiv\left|x_{i}-x_{j}\right| \gg a$, and thus

$$
\left\langle\Pi_{i j}^{h}\right\rangle \sim \frac{e^{i \delta Q x_{i j}}}{\left|x_{i j}\right|^{1 / 2}} .
$$

It then leads to an incommensurate momentum structure at $Q_{0}+\delta Q= \pm 2 k_{f}+2 \pi \times$ integer $\left(k_{f} \equiv(\pi / 2 a)[1-\delta]\right)$ in 1D spin-spin correlation function. Including the time $t$ in Eq. (11) only changes the decay factor $\left|x_{i j}\right|^{-1 / 2}$ to $\left(x_{i j}^{2}\right.$ $\left.-v_{c}^{2} t^{2}\right)^{-1 / 4}$ at long-time limit $\left(v_{c}\right.$ is the Fermi velocity of holon) without modifying the incommensurate position at $2 k_{f}$. The correct power-law decay of the spin-spin correlation function at $J / t \rightarrow 0^{+}$limit is given by

$$
\left\langle S_{i}^{+}(t) S_{j}^{-}(0)\right\rangle \sim \frac{\cos \left(2 k_{f} x_{i j}\right)}{\left(x_{i j}^{2}-v_{s}^{2} t^{2}\right)^{1 / 2}\left(x_{i j}^{2}-v_{c}^{2} t^{2}\right)^{1 / 4}},
$$

where $v_{s}$ is the spinon velocity. Furthermore, the similar phase-string effect also gives rise to a Luttinger-liquid type Fermi-surface singularity in the momentum distribution of electrons at $k_{f}$ with an exponent $1 / 8 .{ }^{14}$ Even though the $2 k_{f}$ incommensurate structure in spin-spin correlations is still connected to the Fermi momentum $k_{f}$ here, a Fermi-liquid type interpretation in terms of the Kohn anomaly is no longer accurate since the electron quasiparticle description is not elementary anymore. A correct picture is that each holon as a topological object carries a spin domain wall with it so that in calculating the large-distance spin-spin correlation function one has to count how many holons in between as each of them contributes a $\pi$ phase-shift as shown in Eq. (7).

\section{B. Incommensurate structure in 2D}

The spin-charge separation in 2D is slightly different from 1D. While in 1D spinons and holons can be treated as decoupled at low-energy and long-wavelength limit, spinons and holons in $2 \mathrm{D}$, though they still belong to independent degrees of freedom, will influence each other through some topological gauge fields even in long-distance limit. For example, spinons are subjected to a lattice gauge field $A_{l m}^{h} \neq 0$ in $2 \mathrm{D}$ in the Hamiltonian ${ }^{14}$ [here $(\mathrm{lm})$ refers to a nearestneighbor link]. As a physical quantity, the spin-spin correlation function defined in Eq. (3) should be gauge invariant, independent of the gauge choice of $A_{l m}^{h}$. This requires $\Pi_{i j}^{h}$ to be able to absorb the gauge phase arising from the spinon propagator under a gauge transformation: $A_{l m}^{h} \rightarrow A_{l m}^{h}+\left(\theta_{l}\right.$ $\left.-\theta_{m}\right)$ and $b_{l \sigma} \rightarrow b_{l \sigma} e^{i \sigma \theta_{l}}$ which leaves the Hamiltonian unchanged. ${ }^{14}$

In fact, $\Pi_{i j}^{h}$ itself can be expressed in terms of a gauge field. Introduce the following relation:

$$
\operatorname{Im} \ln \left(z_{i}-z_{l}\right)-\operatorname{Im} \ln \left(z_{j}-z_{l}\right)=\int_{\Gamma} d \mathbf{r} \cdot \frac{\hat{\mathbf{z}} \times\left(\mathbf{r}-\mathbf{r}_{l}\right)}{\left|\mathbf{r}-\mathbf{r}_{l}\right|^{2}},
$$

where $\Gamma$ is an arbitrary path connecting $i$ and $j$ without crossing the site $l$. In the following we always choose $\Gamma$ as one of the shortest paths between $i$ and $j$ without crossing any lattice site. Then $\Pi_{i j}^{h}$ at $t=0$ can be expressed as

$$
\Pi_{i j}^{h}=e^{i\left(\Phi_{i}^{h}-\Phi_{j}^{h}\right)}=e^{i 2 \int_{\Gamma} d \mathbf{r} \cdot \hat{\mathbf{A}}^{h}(\mathbf{r})},
$$

where

$$
\hat{\mathbf{A}}^{h}(\mathbf{r})=\frac{1}{2} \sum_{l} n_{l} \frac{\hat{\mathbf{z}} \times\left(\mathbf{r}-\mathbf{r}_{l}\right)}{\left|\mathbf{r}-\mathbf{r}_{l}\right|^{2}} .
$$

Similarly the lattice gauge field $A_{l m}^{h}$ in the Hamiltonian ${ }^{14}$ can be also expressed by such a vector potential $\hat{\mathbf{A}}^{h}(\mathbf{r})$ as follows:

$$
A_{l m}^{h}=\int_{m}^{l} d \mathbf{r} \cdot \hat{\mathbf{A}}^{h}(\mathbf{r}) .
$$

Then it is straightforward to verify the gauge invariance of the spin-spin correlation function defined in Eq. (3).

In the mean-field theory, the gauge field $A_{l m}^{h}$ defined at a nearest-neighbor link is treated by smearing the holon number distribution locally. ${ }^{17}$ It is equivalent to replacing $\hat{\mathbf{A}}^{h}(\mathbf{r})$ in Eq. (16) by its continuum version $\mathbf{A}^{h}(\mathbf{r})$ defined by

$$
\mathbf{A}^{h}(\mathbf{r})=\frac{1}{2} \int d^{2} \mathbf{r}^{\prime} \rho^{h}\left(\mathbf{r}^{\prime}\right) \frac{\hat{\mathbf{z}} \times\left(\mathbf{r}-\mathbf{r}^{\prime}\right)}{\left|\mathbf{r}-\mathbf{r}^{\prime}\right|^{2}},
$$

where the holon density $\rho^{h}\left(\mathbf{r}^{\prime}\right)$ is obtained by smearing the holon-number distribution on lattice site, $n_{l}^{h}$, in the continuum space at a scale of $a$. However, if one naively does the same replacement in $\Pi_{i j}^{h}$ [Eq. (14)] for a larger spatial separation of $i$ and $j$, an important effect similar to the singular phase-string effect discussed in 1D would be lost.

Generally, one may define

$$
\Delta \phi_{\Gamma} \equiv 2 \int_{\Gamma} d \mathbf{r} \cdot\left[\hat{\mathbf{A}}^{h}(\mathbf{r})-\mathbf{A}^{h}(\mathbf{r})\right]
$$

and rewrite

$$
\Pi_{i j}^{h}=e^{i 2 \int_{\Gamma} d \mathbf{r} \cdot \mathbf{A}^{h}(\mathbf{r})+i \Delta \phi_{\Gamma}} .
$$

$\Delta \phi_{\Gamma}$ will then keep track of the aforementioned singular effect in passing from $\hat{\mathbf{A}}^{h}(\mathbf{r})$ to $\mathbf{A}^{h}(\mathbf{r})$.

According to Eq. (13), the contribution to $2 \int_{\Gamma} d \mathbf{r} \cdot \hat{\mathbf{A}}^{h}(\mathbf{r})$ from a holon sitting at site $l$ is given by the angle spanned between two straight lines connecting $l-i$ and $l-j$ as shown in Fig. 2(a). The right-hand side (rhs) of Eq. (13) is a continuous function of $\mathbf{r}_{l}$ as long as $\mathbf{r}_{l}$ stays away from the path $\Gamma$ connecting $i$ and $j$. So when $l$ is shifted at a scale of $a$ in the limit of $\left|\mathbf{r}_{i}-\mathbf{r}_{j}\right| \gg a$, the change of the corresponding angle is negligibly small. In this case, taking a continuum limit by neglecting the underlying lattice can be justified. But if $\mathbf{r}_{l}$ crosses the branch cut $\Gamma$, then it is easy to see that the angle defined in Eq. (13) has a $2 \pi$ jump. Figure 2(a) shows a nearest-neighbor lattice link that is across the path $\Gamma$ and 


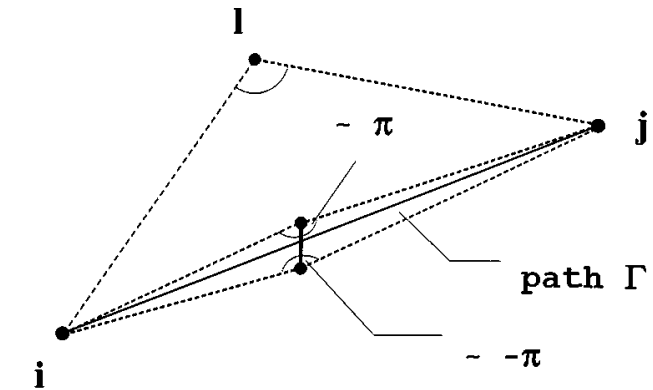

(a)

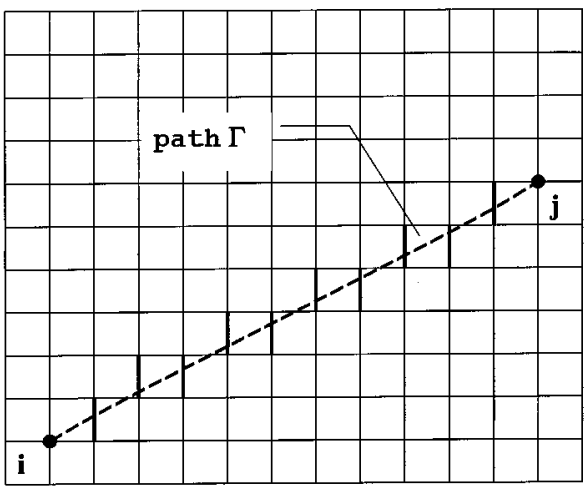

(b)

FIG. 2. (a) The illustration of the angle defined by Eq. (13) at site $l$. Such an angle jumps approximately $2 \pi$ at a nearest-neighbor lattice link (bold one) across the path $\Gamma$ connecting sites $i$ and $j$ in the limit $\left|\mathbf{r}_{i}-\mathbf{r}_{j}\right| \gg a$. (b) The path $\Gamma$ connecting $i$ and $j$ is covered by the nearest-neighbor links along the $\hat{y}$ axis (bold links) in the case that $\left|x_{i}-x_{j}\right| \geqslant\left|y_{i}-y_{j}\right|$.

the value of Eq. (13) changes from $\sim \pi$ to $-\pi$, namely, by $\sim 2 \pi$ for the two lattice sites of the link. It is important to note that in $\Pi_{i j}^{h}$ such a $2 \pi$ change has no effect and thus a holon sitting at either lattice site of the nearest-neighbor link across $\Gamma$ has approximately the same contribution to $\Pi_{i j}^{h}$ by a phase shift $\pi$. On the other hand, $\mathbf{A}^{h}(\mathbf{r})$ is obtained by taking a continuum limit with holon distribution being "coarse grained" at scale of $a$, and in $2 \int_{\Gamma} d \mathbf{r} \cdot \mathbf{A}^{h}(\mathbf{r})$ the contribution from those holons near the path $\Gamma$ within the lattice scale of $a$ will be simply cancelled out due to the opposite signs across $\Gamma$ as shown in Fig. 2(a). Thus, we find

$$
e^{i 2 \int_{\Gamma} d \mathbf{r} \cdot \hat{\mathbf{A}}^{h}(\mathbf{r})} \simeq e^{i 2 \int_{\Gamma} d \mathbf{r} \cdot \mathbf{A}^{h}(\mathbf{r}) \pm i \pi N_{h}(\Gamma),}
$$

where $N_{h}(\Gamma)$ denotes the total number of holons at those nearest-neighboring links across the path $\Gamma$ shown in Fig. 2(b).

So the nonlocal phase shift in $2 \mathrm{D}$ is identified by

$$
\Delta \phi_{\Gamma} \simeq \pm \pi N_{h}(\Gamma) .
$$

As shown in Fig. 2(b), the path $\Gamma$ is always "covered" by nearest-neighbor lattice links along the $\hat{y}$ axis if $\left|x_{i}-x_{j}\right|$ $\geqslant\left|y_{i}-y_{j}\right|$. Noting that each site the average holon number is $\delta$, one has

$$
\left\langle N_{h}(\Gamma)\right\rangle \simeq 2 \delta \frac{\left|x_{i}-x_{j}\right|}{a} .
$$

Similarly, the path $\Gamma$ in Fig. 2(b) can be covered by nearestneighbor lattice links along $\hat{x}$ axis if $\left|x_{i}-x_{j}\right| \leqslant\left|y_{i}-y_{j}\right|$, and the effective holon number along the path $\Gamma$ is estimated to be $2 \delta\left|y_{i}-y_{j}\right| / a$. Thus, two kinds of oscillations in real space are combined to give

$$
\begin{aligned}
e^{i \Delta \phi_{\Gamma} \sim} & \frac{1}{2}\left\{\theta_{i j}^{x} \cos \left[2 \pi \delta / a\left(x_{i}-x_{j}\right)\right]\right. \\
& \left.+\theta_{i j}^{y} \cos \left[2 \pi \delta / a\left(y_{i}-y_{j}\right)\right]\right\},
\end{aligned}
$$

where $\theta_{i j}^{x(y)}=1$ if $\left|x_{i}-x_{j}\right| \geqslant(<)\left|y_{i}-y_{j}\right|$ and otherwise $\theta_{i j}^{x(y)}=0$. by

Finally, the spin-spin correlation function (3) is expressed

$$
\begin{aligned}
\left\langle S_{i}^{+}(t) S_{j}^{-}(0)\right\rangle \simeq & e^{i \mathbf{Q}_{0} \cdot\left(\mathbf{r}_{i}-\mathbf{r}_{j}\right)+i \Delta \phi_{\Gamma}} \\
& \times\left\langle b_{i \uparrow}^{\dagger}(t) b_{i \downarrow}(t)\left(e^{i 2 \int_{\Gamma} d \mathbf{r} \cdot \mathbf{A}^{h}(\mathbf{r})}\right) b_{j \downarrow}^{\dagger}(0) b_{j \uparrow}(0)\right\rangle .
\end{aligned}
$$

The singular phase-string effect is thus retained in the phase factor $e^{i \Delta \phi_{\Gamma}}$ and the gauge-invariant quantity inside $\langle\ldots\rangle$ on the rhs can be now evaluated by the mean-field approximation. In obtaining Eq. (24) we have made approximations by neglecting the fluctuations in $\Delta \phi_{\Gamma}$ itself as well as the temporal dependence of $\Pi_{i j}^{h}(t)$. These effects in 1D lead to an additional decay of the spin-spin correlation function in spatial and temporal space but do not change the incommensurate momentum position. We expect the same thing to happen in 2D where these effects merely cause some extra broadening of magnetic peaks. These broadening effects should become minimal at low-temperature when a Bose condensation of holons occurs, which corresponds to a superconducting condensation ${ }^{17}$ (in the phase-string theory both spinon and holon are bosonic).

Therefore, similar to $1 \mathrm{D}$, the spatial oscillation factor $e^{i \Delta \phi_{\Gamma}}$ in Eq. (24) will lead to incommensurate momentum shifts from $\mathbf{Q}_{0}$ by $\delta \mathbf{Q}=( \pm \bar{\delta} \pi / a, 0)$ and $(0, \pm \bar{\delta} \pi / a)$ according to Eq. (23). [It is noted that $\theta_{i j}^{\eta}$ in Eq. (23) will modify the plane-wave oscillation function but will not change the peak positions at $\delta \mathbf{Q}$.] The incommensurability $\bar{\delta}=2 \delta$ obtained here is in agreement with the experiments discussed in the beginning. Of course, whether such an incommensurate structure can become observable also depends on the broadening effect introduced by the spinon propagator [the average on the rhs of Eq. (24)]. With the singular phase-string effect being explicitly sorted out, such a gauge-invariant quantity can be directly calculated in terms of the mean-field theory developed in Ref. 17. For the sake of compactness, the detailed mathematical manipulation is given in the Appendix. The dynamic spin susceptibility function $\chi^{\prime \prime}(i, j ; \omega)$ is shown in Eq. (A24) (transverse component) and Eq. (A29) ( $z$ component) and is rotationally invariant at the mean-field level.

In Ref. 17, two kinds of mean-field solution have been found for the metallic phase: a uniform phase without a Bose condensation of spinons and an inhomogeneous phase with the Bose condensation of spinons at low temperature. Mathematically they are controlled by the strength of fluctuations in the gauge field $A_{i j}^{h}$ defined in Eq. (A7). Note that the Bose condensation of spinons at half-filling corresponds to a longrange $\mathrm{AF}$ ordering of spins. In the metallic phase, the longrange $\mathrm{AF}$ order is generally absent. But in the spinon Bose 


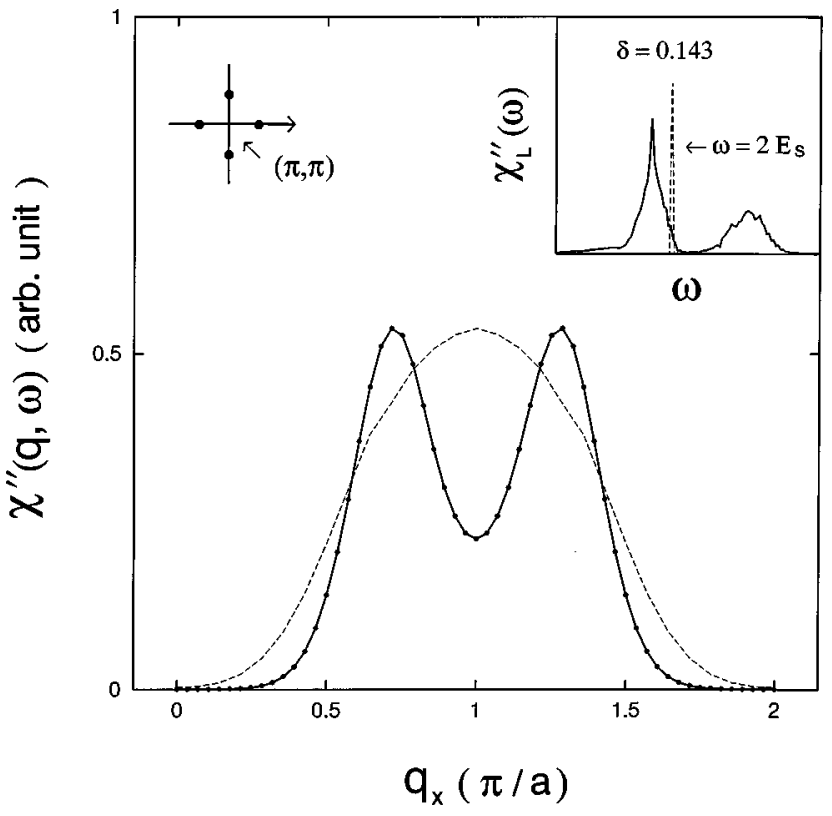

FIG. 3. Dynamic spin susceptibility function $\chi^{\prime \prime}(\mathbf{q}, \omega)$ versus $q_{x}$ (with a fixed $q_{y}$ at $\pi / a$ ) at $\delta=0.143$. Two cases: the dashed curve corresponds to the uniform phase at $\omega=2 E_{s} \sim 0.4 J$ where $\chi_{L}^{\prime \prime}(\omega)$ is peaked (the inset); the solid curve corresponds to the inhomogeneous phase at $\omega \sim 0$. The $\omega$ dependence of $\chi_{L}^{\prime \prime}(\omega)$ in the inset is from Ref. 17. The positions of the incommensurability is illustrated by four full circles at the left top. Note that the maxima of $\chi^{\prime \prime}$ in two cases are scaled to the same value for comparison.

condensation case, a tendency towards the AF ordering is still present as exhibited in a form of inhomogeneity (microscopic phase separation) and is argued to occur usually at smaller doping (underdoped regime). An experimental characterization of those two kinds of metallic phase has been also proposed based on the distinct energy structure ${ }^{17}$ of the local dynamic spin susceptibility function $\chi_{L}^{\prime \prime}(\omega)$, which is illustrated in the inset of Fig. 3 for the same doping concentration $\delta=0.143$ and at $T=0$. The dashed curve corresponds to the uniform phase where at low energy there is only a resonancelike peak at $\omega=2 E_{s} \sim 0.4 J$ ( $J$ is the superexchange coupling constant) and a real spin gap below the peak; the solid curve corresponds to the inhomogeneous phase with a spinon Bose condensation where a double peak is shown with a nonzero but suppressed weight at low energy resembling a pseudogap picture.

We compute the momentum dependence of the dynamic spin susceptibility function corresponding to those two cases with the same parameters. Figure 3 shows $\chi^{\prime \prime}(\mathbf{q}, \omega)$ as the Fourier transformation of $\chi^{\prime \prime}(i, j ; \omega)$ given in the Appendix. The momentum scan is illustrated by the left top inset in Fig. 3 which is along the $q_{x}$ axis at a fixed $q_{y}=\pi / a$, and four full circles denote the incommensurate peak positions at $\mathbf{Q}_{0}$ $+\delta \mathbf{Q}$. First of all, the dashed curve corresponds to the aforementioned uniform-phase case at $\omega=2 E_{s}$. It shows no explicit incommensurate peak splitting because the average in Eq. (24) contributes to a large broadening in momentum space which smears the peak structure composed of four incommensurate peaks. The corresponding resonancelike peak around $\omega=2 E_{s}$ in the inset of Fig. 3 was used to explain ${ }^{17}$ the $41 \mathrm{meV}$ peak in the optimally doped $\mathrm{YBa}_{2} \mathrm{Cu}_{3} \mathrm{O}_{7}$ compound. ${ }^{18}$ A single broad AF peak shown in Fig. 3 is also consistent with the neutron-scattering experiments in such a compound.

The incommensurate peaks at $\mathbf{Q}_{0}+\delta \mathbf{Q}$ explicitly show up in the solid curve (inhomogeneous phase) in Fig. 3 at small $\omega$. Since there is a spinon Bose condensation in this case, the susceptibility function, which has nonzero weight, persists over to low energy until $\omega=0$ (the inset of Fig. 3). In this case, the spin-spin correlation length at small $\omega$ becomes much longer than the uniform-phase case such that the underlying incommensurate structure becomes visible. Experimentally, the incommensurate splitting has been indeed observed at small energy transfer in $\mathrm{La}-\mathrm{Sr}-\mathrm{Cu}-\mathrm{O}$ compounds ${ }^{1-4}$ at the same positions and with the same doping-dependent incommensurability $\bar{\delta}$ shown in Fig. 3. Most recently, a similar incommensurate structure has been also identified in the underdoped $\mathrm{Y}-\mathrm{Ba}-\mathrm{Cu}-\mathrm{O}$ compounds. ${ }^{5}$

\section{Reconciliation of the incommensurability with NMR measurements}

The NMR experiment has served as an another powerful tool in probing the magnetic properties in the cuprates. A combination of NMR and neutron-scattering measurements should provide a more complete picture for the nature of $\mathrm{AF}$ spin-spin correlations in these materials.

The NMR spin-lattice relaxation rate for nuclear spins at planar ${ }^{17} \mathrm{O}$ sites $1 /{ }^{17} T_{1}$ can be expressed in terms of the real-space spin-spin correlations as follows: ${ }^{19-22}$

$$
\frac{1}{{ }^{17} T_{1}} \sim \frac{T}{N} \sum_{i}\left[G_{i i}+\frac{1}{4} \sum_{j=\mathrm{NN}(i)} G_{i j}\right]
$$

where

$$
\left.G_{i j} \equiv \frac{\chi^{\prime \prime}(i, j, \omega)}{\omega}\right|_{\omega \rightarrow 0^{+}} .
$$

According to Eq. (25), $1 /{ }^{17} T_{1}$ only involves real-space spinspin correlations up to nearest-neighboring sites. The spinrelaxation rate for the planar ${ }^{63} \mathrm{Cu}$ nuclear spin can be similarly obtained ${ }^{19-22}$ which involves spin-spin correlations up to next-nearest-neighboring sites. So in the one-component theory ${ }^{19-22}$ of the NMR in cuprates, only short-distance spinspin correlations are relevant.

It has been well known ${ }^{21,22}$ that a sharp commensurate $\mathrm{AF}$ component in $\chi^{\prime \prime}(i, j)$ will have strong cancellation between the on-site and nearest-neighboring-site terms in Eq. (25) due to the staggered factor $(-1)^{i-j}$. So commensurate AF correlations, which presumably are responsible for a so-called non-Korringa temperature behavior in $1 /{ }^{63} T_{1}$, will have much less significant contribution to $1 /{ }^{17} T_{1}$, as has been discussed by many authors, ${ }^{21,22}$ and this is consistent with experimental measurements where $1 /{ }^{17} T_{1}$ shows a much weaker and quite different behavior usually attributed to the non-AF correlations in $\chi^{\prime \prime}$.

However, the neutron-scattering measurements indicate AF correlations in cuprates should be incommensurate in nature as discussed in the previous section. Once the sharp commensurate $\mathrm{AF}$ component is replaced by an incommensurate $\mathrm{AF}$ structure experimentally, it was pointed out ${ }^{10}$ that 
the non-Korringa behavior of $1 / T_{1}$ at ${ }^{63} \mathrm{Cu}$ would usually "leak" to the $1 /{ }^{17} \mathrm{O}$ sites, causing a temperature behavior in $1 /{ }^{17} T_{1}$ which is not consistent with experimental results. This is due to an imperfect cancellation of the AF component of $\chi^{\prime \prime}$ in Eq. (25) after the change from a sharp commensurate to an incommensurate (or a more broadened) magnetic structure around $\mathbf{Q}_{0}$. Indeed, various theories ${ }^{6-8}$ based on the Fermi-surface topology in which the incommensurability arises from the Kohn anomaly have predicted a similar nonKorringa behavior for $1 /{ }^{17} T_{1}$, in contrast to the experimental results. In this sense, incommensurate AF correlations seem inconsistent with the NMR data. In order to resolve this issue, some authors ${ }^{23}$ even have proposed to modify the form of hyperfine coupling as an alternative way out.

But such a paradox can be easily reconciled in the phasestring description without changing the hyperfine interactions which are determined based on the local chemical structure of cuprates and are consistent with the Knight-shift data. ${ }^{19-22}$ In the present theory, the incommensurate structure or spatial modulation, as represented by $e^{i \Delta \phi_{\Gamma}}$ in Eq. (24), has been directly attributed to the phase vortex structure whose singular centers are bound to holons. The resulting incommensurate oscillation in the spin-spin correlation function is only meaningful at a scale much larger than the size of the singular center. As for a nearest-neighbor lattice link $(i j), \Pi_{i j}^{h}$ will simply reduce to

$$
\Pi_{i j}^{h}=e^{i \Phi_{i}^{h}} e^{-i \Phi_{j}^{h}}=e^{i A_{i j}^{h},}
$$

where $A_{i j}^{h}$ is the lattice gauge field given in Eq. (16). In the mean-field approximation $A_{i j}^{h}$ is replaced by $\int_{j}^{i} d \mathbf{r} \cdot \mathbf{A}^{h}(\mathbf{r})$ as discussed before, without causing any additional singular phase shift, namely, $\Delta \phi_{\Gamma}=0$. It is because there can be no holon sitting between two nearest-neighboring spins. Trivially, one also has $\Pi_{i i}^{h}=1$. Thus, $1 /{ }^{17} T_{1}$ in Eq. (25) will simply not be able to "see" any incommensurate phase shift, even though such an incommensurate spatial modulation does exist in large-distance correlations as given by Eq. (23). The latter can be picked up by the neutron-scattering measurement as discussed in the previous section.

Figure 4 shows an example of a non-Korringa behavior of $1 /{ }^{63} T_{1}$ vs a Korringa-like behavior of $1 /{ }^{17} T_{1}$ based on the dynamic spin susceptibility function determined in the Appendix where the uniform (optimal-doping) mean-field solution $^{17}$ is used at $\delta=0.143$. The distinct temperature dependence and the relative size of $1 /{ }^{63} T_{1}$ and $1 /{ }^{17} T_{1}$ here are in accord with the typical experimental data of cuprates. $1 /{ }^{63} T_{1} T$ exhibits a non-Korringa $1 / T$ behavior at high temperature while $1 /{ }^{17} T_{1} T$ remains roughly a constant (Korringa behavior). Note that the decrease of the relaxation rates at low- $T$ is due to the spin gap $\sim E_{s}$ which also decides the superconducting transition temperature $T_{c}$ around the same scale. ${ }^{17}$ One thing we need to emphasize here is that even though in the uniform-phase case the momentum structure in Fig. 3 looks like a commensurate peak, it actually is composed of four incommensurate peaks with stronger broadening, and thus the "leakage" effect would be expected to be even stronger in this case. In fact if we assume the incommensurate spatial modulation (23) to persist into short distance $\sim a$, the non-Korringa temperature dependence will then immediately show up in $1 /{ }^{17} T_{1}$ precisely as pointed out

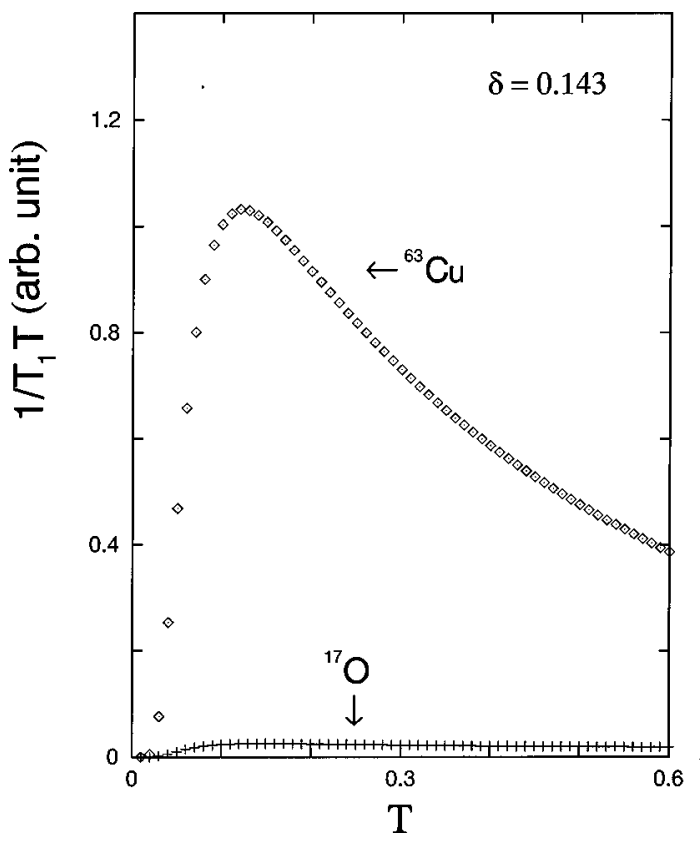

FIG. 4. The contrast of the non-Korringa and Korringa behavior of the NMR spin relaxation rates for planar copper and oxygen nuclear spins for the uniform phase case at $\delta=0.143$.

in Ref.10. Therefore, the origin of the incommensurability from the nonlocal vortex structure of Eq. (2), i.e., the phasestring effect, is indeed crucial to resolve this issue.

\section{DISCUSSIONS}

In the present paper, we have related the magnetic incommensurability to the intrinsic properties of the doped Mott insulator. In the Mott insulator, the spins are the only degrees of freedom not frozen by the strong on-site Coulomb interaction. Holes doped into such a Mott insulator always introduce a phase frustration (phase-string effect) on the spin background by violating the Marshall sign rule. ${ }^{13,14}$ Such a phase-string effect then contributes a unique spatial modulation to the spin-spin correlations as demonstrated in this paper, which is more or less independent of energy and temperature but linearly depends on the doping concentration. In momentum space it leads to an explicit incommensurate splitting of the AF peak in the dynamic spin susceptibility when the spin-correlation length is sufficiently long, in agreement with the neutron-scattering measurements of cuprates in both the peak positions and doping dependence. Furthermore, due to the phase-string origin of this incommensurability, it has been shown that the NMR spinrelaxation rates should not be able to pick up the incommensurability AF correlations at length scales comparable to the lattice constant, and thus conflicting implications of neutron and NMR data can be reconciled within the present framework. Conversely, one may also conclude that the magnetic incommensurability observed in cuprate superconductors provides a direct experimental evidence for the phase-string effect in the doped Mott insulator.

We conclude this paper by making several remarks on some related issues. First of all, we point out that the present magnetic incommensurability is a unique property of metallic phase, which should disappear in the localized regime. 
Recall that the phase-string effect leads to a vortex phase $e^{i \Phi_{i}^{h}}$ emerging in the spin operator (1) which is the reason for the incommensurate spatial modulation. It has been previously discussed ${ }^{17}$ that the same phase $e^{i \Phi_{i}^{h}}$ also causes the disappearance of the AF long-range order. It was argued that only in the hole-localized regime (insulating phase) where the phase-string effect is no longer effective, the phase $e^{i \Phi_{i}^{h}}$ can be compensated by the vortex phase carried by spinons which become vortices in the insulating phase, and the AF long-range order may be recovered. By the same token, the incommensurability induced by $e^{i \Phi_{i}^{h}}$ should be also gone in this localized regime at small doping. This is in accord with the experiments where only a commensurate AF peak has been observed in the insulating phase. ${ }^{4}$

Second, as briefly mentioned in Sec. II A, even though $2 k_{f}$ incommensurate oscillation of the 1D spin-spin correlation function may be viewed as the Kohn anomaly of the Fermi-surface effect, it is not very meaningful because single-electron excitations are no longer elementary and the spin-spin correlation function cannot be reasonably approximated by a "bubble" diagram composed of the singleelectron Green's function without including high-order corrections. Similarly the $2 \mathrm{D}$ magnetic incommensurability in the phase-string formalism does not necessarily exclude the relevance of the Fermi-surface topology. Instead it implies that the latter explanation is no longer accurate as holon and spinon become elementary excitations. Especially the interpretation of the doping-dependent incommensurability and the reconciliation of neutron and NMR measurements can only be naturally realized in this phase-string description of the doped-Mott-insulator.

Finally, we emphasize that the incommensurability discussed in the present paper is due to the topology associated with each individual holon. It has nothing directly to do with the phase separation or charge inhomogeneity at a larger length scale involving many holes as discussed in literature (like the stripe phase ${ }^{24}$ ), including the inhomogeneous (underdoping) metallic regime studied within the phase-string formalism. ${ }^{17}$ Nevertheless, there is still a fundamental connection: all of these may be attributed to the competition between the kinetic energy of holes and superexchange energy of spins and the resulting tendency towards phase separation. The vorticity surrounding each holon may be regarded as a microscopic version of phase separation against a uniform spiral phase. ${ }^{25}$

\section{ACKNOWLEDGMENTS}

The authors acknowledge useful conversations with T. K. Lee, J. X. Li, C. Y. Mou, R. E. Walstedt, and X. G. Wen. The authors would also like to acknowledge the hospitality of the National Center for Theoretical Science in Taiwan where part of the present work was done during their visit. The present work was supported, in part, by the Texas ARP Program No. 3652707 and a grant from the Robert A. Welch foundation, and the State of Texas through the Texas Center for Superconductivity at University of Houston.

\section{APPENDIX: DYNAMIC SPIN SUSCEPTIBILITY FUNCTION}

\section{Mean-field theory}

The $t-J$ model in the phase-string formalism can be written in two terms in the mean-field approximation: ${ }^{17} H_{s}$ $+H_{h} . H_{s}$ governs the spinon degrees of freedom and $H_{h}$ determines the holon degrees of freedom. In the present paper, we mainly focus on the spin magnetic properties decided by $H_{s}$ :

$$
H_{s}=\sum_{m \sigma} E_{m} \gamma_{m \sigma}^{\dagger} \gamma_{m \sigma}+\text { const, }
$$

where $\gamma_{m \sigma}^{\dagger}$ is the creation operator of bosonic spinon elementary excitations. The spinon excitation spectrum $E_{m}$ in this mean-field theory is given by

$$
E_{m}=\sqrt{\lambda_{m}^{2}-\xi_{m}^{2}},
$$

in which $\lambda_{m}$ is defined by

$$
\lambda_{m}=\lambda-\alpha\left|\xi_{m}\right|,
$$

where $\lambda$ is the Lagrangian multiplier to be determined by the condition of the total spin number equal to $N(1-\delta) \quad(N$ is the total number of lattice sites). The coefficient $\alpha$ is a ratio of two energy scales: $\alpha=J_{h} / J_{s}$, in which $J_{h}=\delta t^{\prime}$ describes an effective hopping effect on spinon degrees of freedom with $t^{\prime} \sim J$ and $J_{s}=(J / 2) \Delta^{s}$ with $\Delta^{s}$ being the bosonic RVB order parameter characterizing the present mean-field state. The Lagrangian multiplier $\lambda$ and the RVB order parameter $\Delta^{s}$ are determined by the following mean-field equations, respectively:

$$
2-\delta=\frac{1}{N} \sum_{m} \frac{\lambda_{m}}{E_{m}} \operatorname{coth} \frac{\beta E_{m}}{2}
$$

and

$$
\Delta^{s}=\frac{1}{4 N} \sum_{m} \frac{\xi_{m}^{2}}{J_{s} E_{m}} \operatorname{coth} \frac{\beta E_{m}}{2} .
$$

The spectrum $\xi_{m}$ will be modified by the doping effect through a gauge field $A_{i j}^{h}$ as the eigenvalue of the following equation: ${ }^{17}$

$$
\xi_{m} w_{m \sigma}(i)=-J_{s} \sum_{j=\mathrm{NN}(i)} e^{i \sigma A_{i j}^{h} w_{m \sigma}}(j) .
$$

Here $A_{i j}^{h}$ [with $\left.j=\mathrm{NN}(i)\right]$ is originally defined by the following gauge invariant condition:

$$
\sum_{C} A_{i j}^{h}=\pi N_{C}^{h},
$$

where $C$ is an arbitrary counterclockwise closed path and $N_{C}^{h}$ is the number of holes enclosed by it. In the present meanfield theory, the dynamic effect of $A_{i j}^{h}$ is replaced by a randomness as it comes from an independent degrees of freedom (holons). $N_{C}^{h}$ may be rewritten as $\bar{N}_{C}^{h}+\delta N_{C}^{h}$ with fluctuating $\delta N_{C}^{h}$. Correspondingly $A_{i j}^{h}$ describes a uniform fictitious magnetic field with a random flux fluctuation on the 
top of it. Two kinds of mean-field solutions due to different strengths of the fluctutations are found, ${ }^{17}$ which are distinguished by whether there is a Bose condensation of spinons or not at low temperature. The former case is called the inhomogeneous metallic phase and the latter is called the uniform metallic phase in Ref. 17.

Finally, the Bogoliubov transformation relates the $\gamma$ operator to the spinon operator $b_{i \sigma}$ and is given as follows:

$$
b_{i \sigma}=\sum_{m}\left(u_{m} \gamma_{m \sigma}-v_{m} \gamma_{m-\sigma}^{\dagger}\right) e^{i \sigma \chi_{m}} w_{m \sigma}(i),
$$

where $u_{m}=1 / \sqrt{2}\left(\lambda_{m} / E_{m}+1\right)^{1 / 2}$ and $u_{m}=1 / \sqrt{2}\left(\lambda_{m} / E_{m}\right.$ $-1)^{1 / 2} \operatorname{sgn}\left(\xi_{m}\right)$. The phase factor $e^{i \sigma \chi_{m}}$ is determined up to a change

$$
e^{i \sigma \chi_{m}} \rightarrow-\operatorname{sgn}\left(\xi_{m}\right) \times e^{i \sigma \chi_{m}}
$$

each time when a holon changes sublattices. ${ }^{17}$

\section{Dynamic spin susceptibility}

Let us first consider the transverse component defined in Matsubara representation as follows:

$$
\chi_{+-}\left(i, j ; i \omega_{n}\right)=\int_{0}^{\beta} d \tau e^{i \omega_{n} \tau}\left\langle T_{\tau} S_{i}^{+}(\tau) S_{j}^{-}(0)\right\rangle
$$

Here $\omega_{n}=2 \pi n / \beta$ and

$$
\begin{aligned}
\left\langle T_{\tau} S_{i}^{+}(\tau) S_{j}^{-}(0)\right\rangle= & (-1)^{i-j}\left\langle T_{\tau} b_{i \uparrow}^{\dagger}(\tau) b_{i \downarrow}(\tau)\right. \\
& \left.\times \Pi_{i j}^{h}(\tau) b_{j \downarrow}^{\dagger}(0) b_{j \uparrow}(0)\right\rangle .
\end{aligned}
$$

According to discussions in Sec. II B, the rhs of Eq. (A11) can be expressed by

$$
(-1)^{i-j}\left\langle T_{\tau} b_{i \uparrow}^{\dagger}(\tau) b_{i \downarrow}(\tau) b_{j \downarrow}^{\dagger}(0) b_{j \uparrow}(0) e^{i 2 \int_{\Gamma} d \mathbf{r} \cdot \mathbf{A}^{h}(\mathbf{r})+i \Delta \phi_{\Gamma}}\right\rangle .
$$

In the mean-field approximation one has

$$
\begin{aligned}
\left\langle T_{\tau} b_{i \uparrow}^{\dagger}(\tau) b_{i \downarrow}(\tau) b_{j \downarrow}^{\dagger}(0) b_{j \uparrow}(0)\right\rangle_{s} & \\
= & \left\langle T_{\tau} b_{i \uparrow}^{\dagger}(\tau) b_{j \downarrow}^{\dagger}(0)\right\rangle_{s}\left\langle T_{\tau} b_{j \uparrow}(0) b_{i \downarrow}(\tau)\right\rangle_{s} \\
& +\left\langle T_{\tau} b_{i \uparrow}^{\dagger}(\tau) b_{j \uparrow}(0)\right\rangle_{s}\left\langle T_{\tau} b_{i \downarrow}(\tau) b_{j \downarrow}^{\dagger}(0)\right\rangle_{s},
\end{aligned}
$$

where $\langle\ldots\rangle_{s}$ denotes the average over the spinon degrees of freedom. According to the mean-field theory outlined in the above section, one has

$$
\begin{aligned}
b_{i \sigma}^{\dagger}(\tau) & =e^{H_{s} \tau} b_{i \sigma}^{\dagger} e^{-H_{s} \tau} \\
& =\sum_{m}\left(u_{m} \gamma_{m \sigma}^{\dagger} e^{E_{m} \tau}-v_{m} \gamma_{m-\sigma} e^{-E_{m} \tau}\right) w_{m \sigma}^{*}(i) e^{-i \sigma \chi_{m}} .
\end{aligned}
$$

$$
\begin{aligned}
\left\langle T_{\tau} b_{i \sigma}^{\dagger}(\tau) b_{j-\sigma}^{\dagger}(0)\right\rangle_{s} \\
=-\sum_{m} u_{m} v_{m}\left[w_{m \sigma}^{*}(i) w_{m \sigma}(j)\right] \\
\quad \times e^{-i \sigma \Delta \chi_{m}}\left(e^{E_{m} \tau}\left\langle\gamma_{m \sigma}^{\dagger} \gamma_{m \sigma}\right\rangle_{s}+e^{-E_{m} \tau}\left\langle\gamma_{m-\sigma} \gamma_{m-\sigma}^{\dagger}\right\rangle_{s}\right),
\end{aligned}
$$

at $\tau>0$ where $e^{-i \sigma \Delta \chi_{m}}$ is introduced to incorporate the phase-shift effect (A9). $e^{-i \sigma \Delta \chi_{m}}$ may be determined based on how many exchanges there are between the spinon and background holons. The spinon will acquire a phase shift $\pm \pi$ for $\xi_{m}>0$ due to $-\operatorname{sgn}\left(\xi_{m}\right)=-1$ each time it exchanges positions with a holon. Thus, if $N_{i j}^{h}$ denotes the number of holons encountered and exchanged with the involved spinon on the shortest path $\Gamma$ connecting $i$ and $j$, the total phase shift acquired by the spinon at $\xi_{m}>0$ is $\pm \pi N_{i j}^{h} \simeq \Delta \phi_{\Gamma}$. Then we may write down

$$
e^{-i \sigma \Delta \chi_{m}}= \begin{cases}1, & \text { if } \xi_{m}<0, \\ (-1)^{N_{i j}^{h},} & \text { if } \xi_{m}>0 .\end{cases}
$$

It is noted that for each $m$ with $\xi_{m}<0$, one always can find a state $\bar{m}$ with $\xi_{m}=-\xi_{m}>0$ with a wave function

$$
w_{\bar{m} \sigma}(i)=(-1)^{i} w_{m \sigma}(i)
$$

according to Eq. (A6). Then in terms of Eqs. (A16) and (A17) we finally have (at $\tau>0$ )

$$
\begin{aligned}
\left\langle T_{\tau} b_{i \sigma}^{\dagger}(\tau) b_{j-\sigma}^{\dagger}(0)\right\rangle_{s}= & {\left[(-1)^{\left.i-j+N_{i j}^{h}-1\right]}\right.} \\
& \times \sum_{m}{ }^{\prime} u_{m} v_{m}\left[w_{m \sigma}^{*}(i) w_{m \sigma}(j)\right] \\
& \times\left\{n\left(E_{m}\right) e^{E_{m} \tau}+\left[1+n\left(E_{m}\right)\right] e^{-E_{m} \tau}\right\},
\end{aligned}
$$

where the summation $\Sigma_{m}^{\prime}$ only runs over those states with $\xi_{m}<0$. Similarly, one can also determine

$$
\begin{aligned}
\left\langle T_{\tau} b_{i \sigma}^{\dagger}(\tau) b_{j \sigma}(0)\right\rangle_{s}= & {\left[(-1)^{\left.i-j+N_{i j}^{h}+1\right]}\right.} \\
& \times \sum_{m}{ }^{\prime}\left[w_{m \sigma}^{*}(i) w_{m \sigma}(j)\right]\left\{u_{m}^{2} n\left(E_{m}\right) e^{E_{m} \tau}\right. \\
& \left.+v_{m}^{2}\left[1+n\left(E_{m}\right)\right] e^{-E_{m} \tau}\right\},
\end{aligned}
$$

as well as other averages in Eq. (A13).

Then after integrating out $\tau$ in Eq. (A10) and identifing $(-1)^{N_{i j}^{h}}$ with $e^{i \Delta \phi_{\Gamma}}$ in the operator form, one obtains

$$
\begin{aligned}
\chi_{+-}\left(i, j ; i \omega_{n}\right) \simeq & \chi_{+-}^{(-)}\left(i, j ; i \omega_{n}\right) \\
& +(-1)^{i-j} e^{i \Delta \phi_{\Gamma}} \cdot \chi_{+-}^{(+)}\left(i, j ; i \omega_{n}\right),
\end{aligned}
$$

where

Then by noting $w_{m-\sigma}^{*}=w_{m \sigma}$ one finds 


$$
\begin{aligned}
\chi_{+-}^{( \pm)}\left(i, j ; i \omega_{n}\right)= & \sum_{m m^{\prime}}{ }^{\prime} K_{m m^{\prime}}^{+-}(i, j) \\
& \times\left[\left(p_{m m^{\prime}}^{ \pm}\right)^{2} \frac{n\left(E_{m^{\prime}}\right)-n\left(E_{m}\right)}{i \omega_{n}+E_{m}-E_{m^{\prime}}}\left(l_{m m^{\prime}}^{ \pm}\right)^{2}\right. \\
& \times\left[1+n\left(E_{m}\right)+n\left(E_{m^{\prime}}\right)\right] \\
& \left.\times \frac{1}{2}\left(\frac{1}{i \omega_{n}+E_{m}+E_{m^{\prime}}}-\frac{1}{i \omega_{n}-E_{m}-E_{m^{\prime}}}\right)\right],
\end{aligned}
$$

with

$$
\begin{aligned}
K_{m m^{\prime}}^{+-}(i, j)= & 2\left[e^{i \int_{\Gamma} d \mathbf{r} \cdot \mathbf{A}^{h}} w_{m \uparrow}^{*}(i) w_{m \uparrow}(j)\right] \\
& \times\left[e^{i \int_{\Gamma} d \mathbf{r} \cdot \mathbf{A}^{h}} w_{m \downarrow}^{*}(j) w_{m \downarrow}(i)\right] \\
= & \sum_{\sigma}\left[e^{i \sigma \int_{\Gamma} d \mathbf{r} \cdot \mathbf{A}^{h}} w_{m \sigma}^{*}(i) w_{m \sigma}(j)\right] \\
& \times\left[e^{i \sigma \int_{\Gamma} d \cdot \mathbf{A}^{h}} w_{m-\sigma}^{*}(j) w_{m-\sigma}(i)\right] .
\end{aligned}
$$

In obtaining the last line, the symmetry $w_{m \sigma}^{*}=w_{m-\sigma}$ has been used. The coherent factors, $p_{m m^{\prime}}^{ \pm}$and $l_{m m^{\prime}}^{ \pm}$are defined by

$$
\begin{gathered}
p_{m m^{\prime}}^{ \pm}=u_{m} u_{m^{\prime}} \pm v_{m} v_{m^{\prime}}, \\
l_{m m^{\prime}}^{ \pm}=u_{m} v_{m^{\prime}} \pm v_{m} u_{m^{\prime}} .
\end{gathered}
$$

Finally, the dynamic spin susceptibility function $\chi_{+-}^{\prime \prime}(i, j ; \omega)$ can be obtained as the imaginary part of $\chi_{+-}$after an analytic continuation $i \omega_{n} \rightarrow \omega+i 0^{+}$is made:

$$
\chi_{+-}^{\prime \prime}(i, j ; \omega)=\Phi_{+-}^{(-)}(i, j ; \omega)+(-1)^{i-j} e^{i \Delta \phi_{\Gamma}} \cdot \Phi_{+-}^{(+)}(i, j ; \omega),
$$

where

$$
\begin{aligned}
\Phi_{+-}^{( \pm)}(i, j ; \omega)= & \frac{\pi}{2} \sum_{m m^{\prime}}{ }^{\prime} K_{m m^{\prime}}^{+-}(i, j)\left\{\left[1+n\left(E_{m}\right)+n\left(E_{m^{\prime}}\right)\right]\right. \\
& \times\left(l_{m m^{\prime}}^{ \pm}\right)^{2} \operatorname{sgn}(\omega) \delta\left(|\omega|-E_{m}-E_{m^{\prime}}\right) \\
& +2\left[n\left(E_{m}\right)-n\left(E_{m^{\prime}}\right)\right]\left(p_{m m^{\prime}}^{ \pm}\right)^{2} \\
& \left.\times \delta\left(\omega+E_{m}-E_{m^{\prime}}\right)\right\} .
\end{aligned}
$$

The longitudinal spin susceptibility function can be similarly obtained:

$$
\begin{aligned}
\chi_{z z}\left(i, j ; i \omega_{n}\right) \equiv & \int_{0}^{\beta} d \tau e^{i \omega_{n}}\left\langle T_{\tau} S_{i}^{z}(\tau) S_{j}^{z}(0)\right\rangle \\
= & \chi_{z z}^{(-)}\left(i, j ; i \omega_{n}\right)+(-1)^{i-j} \\
& \times e^{i \Delta \phi_{\Gamma}} \cdot \chi_{z z}^{(+)}\left(i, j ; i \omega_{n}\right),
\end{aligned}
$$

where $S_{i}^{z}=\sum_{\sigma} \sigma b_{i \sigma}^{\dagger} b_{i \sigma}$ is used and

$$
\begin{aligned}
\chi_{z z}^{( \pm)}\left(i, j ; i \omega_{n}\right)= & \frac{1}{2} \sum_{m m^{\prime}}{ }^{\prime} K_{m m^{\prime}}^{z z}(i, j) \\
& \times\left[\left(p_{m m^{\prime}}^{ \pm}\right)^{2} \frac{n\left(E_{m^{\prime}}\right)-n\left(E_{m}\right)}{i \omega_{n}+E_{m}-E_{m^{\prime}}}\right. \\
& +\left(l_{m m^{\prime}}^{ \pm}\right)^{2}\left[1+n\left(E_{m}\right)+n\left(E_{m^{\prime}}\right)\right] \\
& \left.\times \frac{1}{2}\left(\frac{1}{i \omega_{n}+E_{m}+E_{m^{\prime}}}-\frac{1}{i \omega_{n}-E_{m}-E_{m^{\prime}}}\right)\right]
\end{aligned}
$$

with

$$
\begin{aligned}
K_{m m^{\prime}}^{z z}(i, j) \equiv & \sum_{\sigma}\left[e^{i \sigma \int_{\Gamma} d \mathbf{r} \cdot \mathbf{A}^{h}} w_{m \sigma}^{*}(i) w_{m \sigma}(j)\right] \\
& \times\left[e^{-i \sigma \int_{\Gamma} d \mathbf{r} \cdot \mathbf{A}^{h}} w_{m^{\prime} \sigma}^{*}(j) w_{m^{\prime} \sigma}(i)\right],
\end{aligned}
$$

where the phase factors $e^{ \pm i \sigma \int_{\Gamma} d \mathbf{r} \cdot \mathbf{A}^{h}}$ are introduced to explicitly show the gauge invariance of $K_{m m^{\prime}}^{z z}$. Generally, one expects the gauge-invariant quantity $e^{i \sigma \int_{\Gamma} d \mathbf{r} \cdot \mathbf{A}^{h}} w_{m \sigma}^{*}(i) w_{m \sigma}(j)$ to be independent of $\sigma$ or to be real, which in turn means $K_{m m^{\prime}}^{z z}=K_{m m^{\prime}}^{+-}$. Consequently, the rotational invariance of spin-spin correlations is retained. The corresponding dynamic spin susceptibility function is given by

$$
\chi_{z z}^{\prime \prime}(i, j ; \omega)=\Phi_{z z}^{(-)}(i, j ; \omega)+(-1)^{i-j} e^{i \Delta \phi_{\Gamma}} \Phi_{z z}^{(+)}(i, j ; \omega),
$$

where

$$
\begin{aligned}
\Phi_{z z}^{( \pm)}(i, j ; \omega)= & \frac{\pi}{2} \sum_{m m^{\prime}}{ }^{\prime} K_{m m^{\prime}}^{z z}(i, j)\left\{\left[1+n\left(E_{m}\right)+n\left(E_{m^{\prime}}\right)\right]\right. \\
& \times\left(l_{m m^{\prime}}^{ \pm}\right)^{2} \operatorname{sgn}(\omega) \delta\left(|\omega|-E_{m}-E_{m^{\prime}}\right) \\
& +2\left[n\left(E_{m}\right)-n\left(E_{m^{\prime}}\right)\right]\left(p_{m m^{\prime}}^{ \pm}\right)^{2} \\
& \left.\times \delta\left(\omega+E_{m}-E_{m^{\prime}}\right)\right\} .
\end{aligned}
$$

${ }^{1}$ G. Shirane, R. J. Birgneau, Y. Endoh, P. Gehring, M. A. Kastner, K. Kitazawa, H. Kojima, I. Tanaka, T. R. Thurston, and K. Yamada, Phys. Rev. Lett. 63, 330 (1989).

${ }^{2}$ S-W. Cheong, G. Aeppli, T. E. Mason, H. Mook, S. M. Hayden, P. C. Canfield, Z. Fisk, K. N. Clausen, and J. L. Martinez, Phys. Rev. Lett. 67, 1791 (1991).
${ }^{3}$ T. E. Mason, G. Aeppli, and H. A. Mook, Phys. Rev. Lett. 68, 1414 (1992).

${ }^{4}$ K. Yamada, C. H. Lee, K. Kurahashi, J. Wada, S. Wakimoto, S. Ueki, H. Kimura, Y. Endoh, S. Hosoya, G. Shirane, R. J. Birgeneau, M. Greven, M. A. Kastner, and Y. J. Kim, Phys. Rev. B 57, 6165 (1998). 
${ }^{5}$ H. A. Mook, P. Dai, R. D. Hunt, and F. Doğan, cond-mat/9712326 (unpublished).

${ }^{6}$ Q. Si, Y. Zha, K. Levin, and J. P. Lu, Phys. Rev. B 47, 9055 (1993).

${ }^{7}$ P. B. Littlewood, J. Zaanen, G. Aeppli, and H. Monien, Phys. Rev. B 48, 487 (1993).

${ }^{8}$ T. Tanamoto, H. Kohno, and H. Fukuyama, J. Phys. Soc. Jpn. 62, 717 (1993); 63, 2739 (1994).

${ }^{9}$ P. W. Anderson, Science 235, 1196 (1987); P. W. Anderson, The Theory of Superconductivity in the High $T_{c}$ Cuprates (Princeton University Press, Princeton, 1997).

${ }^{10}$ R. E. Walstedt, B. S. Shastry, and S-W. Cheong, Phys. Rev. Lett. 72, 3610 (1994), and references therein.

${ }^{11}$ S. Chakravarty, B. I. Halperin, and D. R. Nelson, Phys. Rev. Lett. 60, 1057 (1988).

${ }^{12}$ See, A. V. Chubukov, S. Sachdev, and J. Ye, Phys. Rev. B 49, 11 919 (1994), and the references therein.

${ }^{13}$ D. N. Sheng, Y. C. Chen, and Z. Y. Weng, Phys. Rev. Lett. 77, 5102 (1996).

${ }^{14}$ Z. Y. Weng, D. N. Sheng, Y. C. Chen, and C. S. Ting, Phys. Rev. B 55, 3894 (1997).

${ }^{15}$ S. Liang, B. Doucot, and P. W. Anderson, Phys. Rev. Lett. 61, 365 (1988).
${ }^{16}$ D. P. Arovas and A. Auerbach, Phys. Rev. B 38, 316 (1988); A. Auerbach and D. P. Arovas, Phys. Rev. Lett. 61, 617 (1988).

${ }^{17}$ Z. Y. Weng, D. N. Sheng, and C. S. Ting, Phys. Rev. Lett. 80, 5401 (1998); Phys. Rev. B 59, 8943 (1999).

${ }^{18}$ M. A. Mook, Yethiraj, G. Aeppli, T. E. Mason, and T. Armstrong, Phys. Rev. Lett. 70, 3490 (1993); H. F. Fong, B. Keimer, P. W. Anderson, D. Renzik, F. Doğan, and I. A. Aksay, ibid. 75, 316 (1995).

${ }^{19}$ F. Mila and T. M. Rice, Physica C 17, 561 (1989).

${ }^{20}$ B. S. Shastry, Phys. Rev. Lett. 63, 1288 (1989).

${ }^{21}$ N. Bulut, D. Hone, D. J. Scalapino, and N. E. Bickers, Phys. Rev. Lett. 64, 2723 (1990).

${ }^{22}$ A.J. Millis, H. Monien, and D. Pines, Phys. Rev. B 42, 167 (1990).

${ }^{23}$ Y. Zha, V. Barzykin, and D. Pines, Phys. Rev. B 54, 7561 (1996).

${ }^{24}$ J. M. Tranquanda et al., Nature (London) 375, 561 (1995); Phys. Rev. B 54, 7489 (1996); Phys. Rev. Lett. 78, 338 (1997).

${ }^{25}$ B. I. Shraiman and E. D. Siggia, Phys. Rev. Lett. 62, 1564 (1989); 61, 467 (1988); C. Jayaprakash et al., Phys. Rev. B 40, 2610 (1989); D. Yoshioka, J. Phys. Soc. Jpn. 58, 1516 (1989); C. L. Kane et al., Phys. Rev. B 41, 2653 (1990); Z. Y. Weng, Phys. Rev. Lett. 66, 2156 (1991). 